\title{
VOLTAGE COLLAPSE AVOIDANCE IN POWER SYSTEMS: A RECEDING HORIZON APPROACH
}

\author{
S. A. Attia, M. Alamir and C. Canudas de Wit \\ Laboratoire d'Automatique de Grenoble \\ Domaine Universitaire BP46 \\ 38402 Saint Martin d'Hères \\ France \\ e-mail:attia@ieee.org,Mazen.Alamir@inpg.fr,carlos.canudas-de-wit@lag.ensieg.inpg.fr
}

\begin{abstract}
In this paper, a receding horizon control approach is developed for voltage stabilization in power systems. The primary focus is on a benchmark system proposed in the context of a European project. The system is termed hybrid due to the finite set of control inputs, state automata description of the voltage primary controller and their interactions with the load nonlinear continuous dynamics. Moreover, the network topology imposes hard constraints on the states. All these make the control problem quite challenging. For different realistic scenarios, the proposed control algorithm is shown to stabilize the bus voltages at acceptable levels. The computational demand fits the real time allowed slot, making the scheme attractive for large scale applications as an area central controller.
\end{abstract}

Key Words: Hybrid systems, predictive control, voltage stability, benchmark problem

\section{INTRODUCTION}

Besides the challenging control problems posed by the large scale and distributed character of power systems, an important hybrid aspect is put forward by the discrete continuous interactions both at the physical and control levels. Consequently, power systems are considered as paradigms of hybrid systems, see e.g., [1]. The theoretical foundations for hybrid systems has reached a moderate degree of maturity [2][4] this include both modelling and stability frameworks, but to date no general controller synthesis methodologies are available, it seems that this is system class and application's related.

In the last decade, the power community has shown a great interest in systematic design methods for assessing stability in power networks. Voltage instability is undeniably one of the most inconstant and costly failures, for instance, the 14 August 2003 blackout in North America cost between US $\$ 4$ billion and $\$ 6$ billion [5]. Basic material on voltage stability philosophical concepts may be found in the recent surveys [6]-[7]. The interested reader may also consult [8]-[9] for general definitions and basic problems.

Much of the work reported on voltage stability assessment relies on heuristics. Where basically, knowledge of the system and experience gained by the designers is put forward in schemes such those developed in [10] where heuristics based load shedding strategies are compared with branch and bound based ones. The multi step design consist in time domain simulation for generating training scenarios. An optimization soundstage is then carried out to minimize the amount of load shedding with respect to the value found in the training set. Another family of methods rely on computing a security margin or distance to voltage collapse, see e.g., [11] for a survey. Based on these measures, sensitivities with respect to the different control actions are then computed. These provide directions towards the control must be updated, this is in general followed by a constrained multiple optimization stage for dispatching the different control actions, see e.g., [12] where a coordinated controller is synthesized by taking as a margin the minimum distance between the operating point and bifurcation boundary as measured in the parameter space. In the same spirit, [13] use a quasi steady state simulation approach. A simple formula is then proposed to update the different control actions based on off line bus ranking, see also [14] and [15] for other variants. In [16] a clear distinction is made between the different control actions. And a scheme is derived where corrective 
and preventive control strategies interact, the first is used in extreme contingencies (loss of system solvability) while the latter is for enhancing the system stability margin.

The method developed in this paper fall into the category where knowledge of the system is reduced at its basic. Only a simulation model is indeed needed. This model should incorporate at least the dominant features i.e., the load dynamics since voltage collapse is driven by these dynamics. These approaches include mainly methods based on predicting and analyzing sensitivities of the system trajectories see e.g., [17] where this is discussed in the more general framework of hybrid systems. See also [18] where a predictive control approach is investigated. Based on evolutionary programming techniques, an optimization stage is carried out over the space of all control inputs. The continuous set point inputs as well as the discrete ones i.e., the capacitor banks and load shedding mechanisms. The predictive control approach is also exploited in the work [19] where an improved depth first search method from the Artificial Intelligence community is used as an alternative to further reduce the combinatorics. Material on predictive control can be found in [20]-[23]. The aim of the present paper is to explore a particular controller design methodology for voltage collapse avoidance in power systems with emphasis on a particular benchmark problem [24]. This is a step towards a fully coordinated decentralized solution in the spirit of [25], where the focus is on area central controller. We will not deal with higher level controllers used to coordinate areas controllers, indeed we believe that at a higher level an expert system with advanced heuristics shall be used, the interested reader may consult the multi agent approaches e.g., [26][27]. The controller developed is based on an efficient open loop parametrization that drastically reduces the combinatorics associated to a prediction based methodology. Then a terminal inequality constraint is imposed on the bus voltages, discrimination of "good admissible controls" from the "bad ones" thus follows. Based on that, an optimization stage is carried out to extract the optimal open loop control sequence. The first component of which is applied and the process is repeated in a receding horizon fashion [20]. The controller computational burden is shown to be compatible with the available computation time for a large number of prediction horizons.

The paper is organized as follows: section 2 presents a brief analysis the benchmark power system under consideration. Section 3 is devoted to the predictive control approach. In section 4 some validating simulations and algorithm computational times are reported. Finally, some conclusions and future orientations are given in section 5 .

\section{POWER SYSTEM ANALYSIS}

The power system under study is proposed as a benchmark by ABB [24] in the framework of the European Project Control \& Computation. This in order to illustrate control strategies on hybrid systems. The power system consists in a four node transmission system as shown in figure 1, see also [24] for a complete description

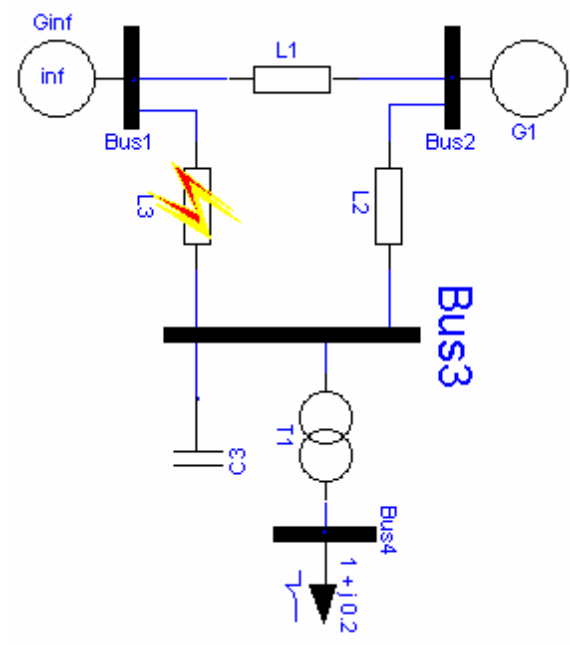

Figure 1. The ABB transmission test case

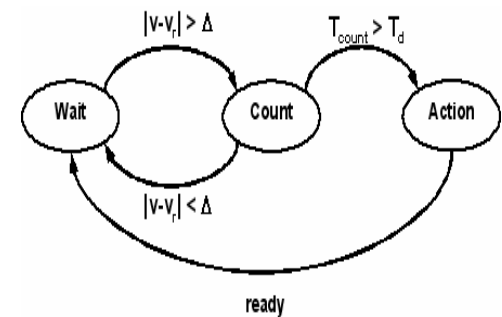

Figure 2. The OLTC finite state machine model 
The system components are as follow

- An infinite bus which represents the rest of the network. The system under consideration can not influence appreciably the rest of the power system network. This bus is also used as a slack in the modelling phase.

- A generator $G_{1}$ which is modelled as a steady version of the three axis model. This is a valid model since the dynamics of interest are well beyond (slower) the angular frequency.

- Three transmission lines modelled as pure reactance. And refereed as $X_{i j}$ where the subscripts $i$ and $j$ refer respectively to the departure and arrival buses. It is clear that $X_{i j}=X_{j i}$ since the path is unique. Next, it is assumed that only the variation of $X_{13}$ is allowed due to a fault occurrence. This variation is considered as a measurable disturbance.

- A reactive power source represented by the capacitor banc $B$. This is actually a control input to the system.

- A transformer equipped by an On Load Tap Changer (OLTC). Which can regarded as a transformer with a controlled turn ratio $n$. The OLTC is modelled as a finite state machine, see figure. The operation of which is as follows, the system remains in the state wait as long as the voltage deviation $\left(\left|v_{r}-v\right|\right)$ is less than a specified dead zone $\Delta$. When the limit is exceeded, a transition to state count occurs. Upon entering count, a timer is started and is kept running until either it reaches the delay time $T_{d}$ causing a transition to the state action or the voltage deviation becomes less than the dead zone causing a transition to the state wait and reset of the timer. When entering the state action, an update of the turn ratio is operated

$$
n^{+}=\left\{\begin{array}{cccc}
n^{-}+d n & v_{r}-v>\Delta & \wedge & n^{-}<n_{\max } \\
n^{-}-d n & v_{r}-v<-\Delta & \wedge & n^{-}>n_{\min }
\end{array}\right.
$$

where the superscript + and - represent respectively the instants just before and after the instant of update. $n_{\min }$ and $n_{\max }$ is the maximal and minimal allowed values. Once the tap operation completed, the control system receives a ready signal and the control system returns to state wait. It is important here to recall that the control input to the OLTC is the voltage reference $v_{r}(\cdot)$.

- A nonlinear load with recovery dynamics described by the following first order differential equations [24]

$$
\begin{aligned}
& \dot{x}_{p}=-\frac{x_{p}}{T_{p}}+P_{0}\left(v^{\alpha}-v^{\alpha}\right) \\
& \dot{x}_{q}=-\frac{x_{q}}{T_{q}}+Q_{0}\left(v^{\beta_{s}}-v^{\beta_{t}}\right)
\end{aligned}
$$

and the following output equations representing respectively the absorbed active and reactive power

$$
\begin{aligned}
& P=(1-k)\left(\frac{x_{p}}{T_{p}}+P_{0} v^{\alpha_{t}}\right) \\
& Q=(1-k)\left(\frac{x_{q}}{T_{q}}+Q_{0} v^{\beta_{t}}\right)
\end{aligned}
$$

where $x=\left[x_{p} x_{q}\right]^{T}$ represent the load internal states and $P_{0}$ and $Q_{0}$ the active and reactive power steady state values. The others parameters can be found in [24]. The variable $k$ represent the load shedding percentage and is the third control input.

The model of the power system can be written as a DAE 


$$
\begin{aligned}
\dot{x} & =f(x, y, p) \\
0 & =g(x, y, u, p)
\end{aligned}
$$

where the vector differential equation represents the load dynamics, $x$ are the internal states, $y$ the algebraic variables (bus voltages), for the example under study $y$ regroups the bus voltages, $p$ are power system parameters for instance, the line reactances $X_{i j}, u$ represents the control vector and regroups devices such capacitor bank, load shedding, tap changer control and AVR stepoints.

By using a coordinated action of the tap changer, the capacitor bank and the load shedding. The designed controller should be able to stabilize and restore acceptable equilibria for the power system. In figure 3 are plotted the bus voltages against the line reactance. As shown, the case where $X_{13}^{n}=0.5 p . u$ corresponds to the nominal steady state solution. The solutions drop when the value of $X_{13}$ exceeds 1.0 p.u and above $X_{13}^{b}=1.02$ p.u no steady state solutions are feasible, so that for this value and beyond the power system can not be solved (loss of the DAE system's solvability). This case corresponds in fact to a singularity induced bifurcation [28]. This is dealt with in this case study. Recall that when the power system is in fault, the $X_{13}$ reactance passes from $0.5 p . u$ its nominal value to $X_{13}^{f}=1.5 p . u$ which is well beyond $X_{13}^{b}=1.02$ p.u.

It is a well known fact, that the steady state power demand ( $P_{0}$ in equations (2)-(3)) is a slowly but time varying parameter. Figure 4 shows the evolution of the steady state values of the voltages as a function of $P_{0}$ where no fault is assumed. As one can see, beyond $P_{0}^{b}=1.53 p . u$ no solutions do exist for the voltages. This can be seen as an active power limit that can be drawn from the power system. This is to be connected with the $P$ - $V$ curves [29] where only the stable region is plotted. The same quantity is plotted in figure 5 with respect to variations in the steady state reactive power demand $Q_{0}$ in equations (2)-(3). The system loses its solutions when $Q_{0}^{b}=0.89$ p.u and above which is a limit reactive power that can be absorbed by the load without inducing a voltage collapse.

In figure 6 is plotted the steady state voltage solution versus the capacitor bank and load shedding $(100 \times k$ in equations (3)). This plot shows that in order to restore an equilibria about $0.3 p . u$ of the capacitor must be activated.

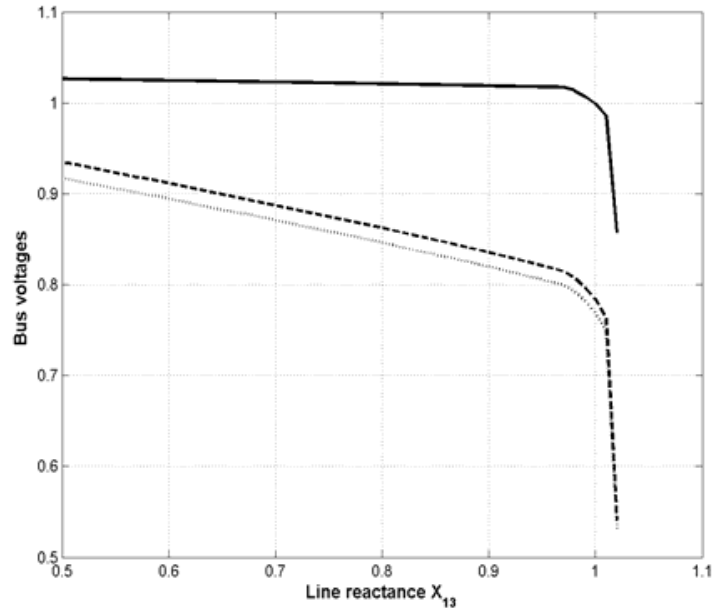

Figure 3. Bus voltages steady state values as a function of the line reactance.

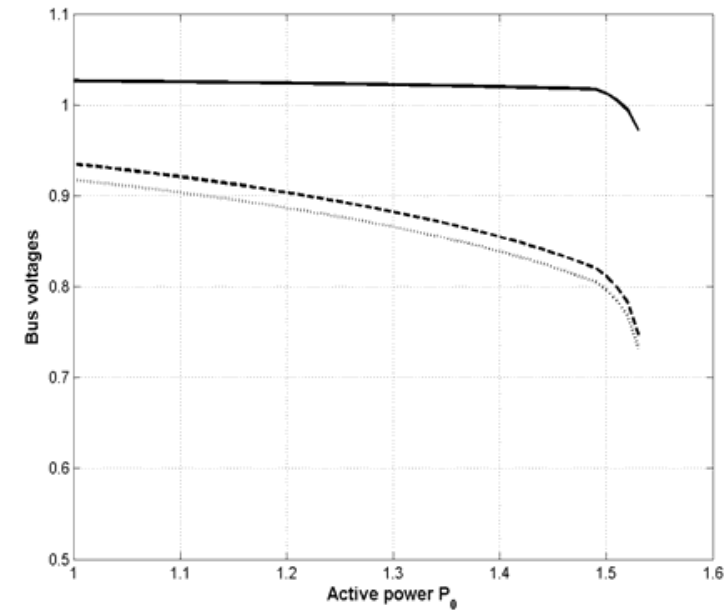

Figure 4. Bus voltages steady state values as a function of the active power. 


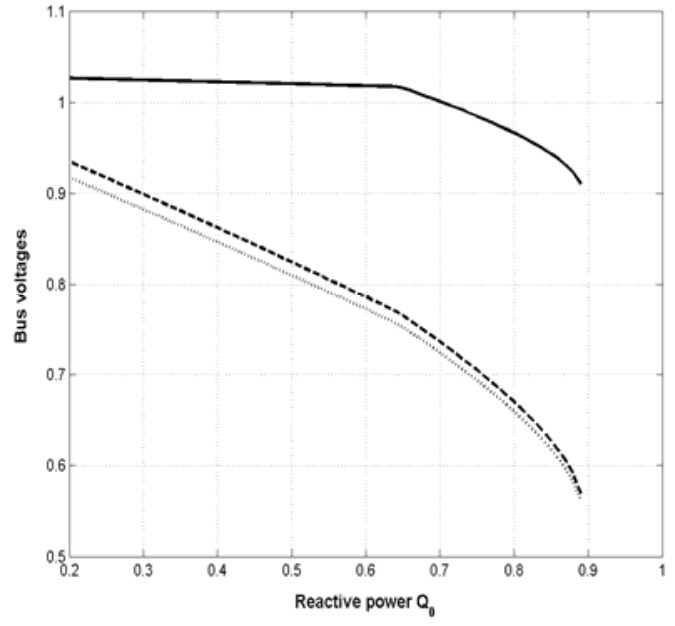

Figure 5. Bus voltages steady state values as a function of the reactive power.



Figure 6. Load voltage for variable capacitor and load shedding.

As we shall see later, one can not rely only on static analysis to derive control policies. This is the optimal power flow based methods (purely static setting) as known in literature see e.g., [30]. This methodology can be satisfactory, when the static analysis's derived controls are applied as soon as the fault appears, or the case where some advanced heuristics are introduced leading to efficient timing of the different actions. The voltage collapse as seen in this contribution is a dynamic process needing efficient timing of the actions and thus a closed loop control strategy.

More expressively, the control objectives are to fulfill the following requirements

- Stabilize all voltages at values above 0.9 p.u following the fault.

- Minimize the amount of load shedding applied.

- Keep the voltage at bus 4 close to 1 p.u while minimizing the amount of capacitor control to do so.

Before we proceed further, let us denote the vector of bus voltages $\left[\begin{array}{ll}v_{2} & v_{3} \\ v_{4}\end{array}\right]^{T}$ as $y$. We have the following constraints on the control inputs. The load shedding percentage $k$ belongs to the following discrete set with $k_{\max }=0.15$

$$
k \in \mathrm{K}:=\left\{0.00,0.05, \cdots_{\max }\right\}
$$

The capacitor banc belongs to the following discrete set of admissible capacitors with $b_{\max }=0.3$

$$
b \in \mathrm{B}=\left\{0.0,0.1, \ldots b_{\max }\right\}
$$

It is worth noting that because of the tap changer dynamics, the transformer ratio $n$ cannot be rigourously considered as a control variable. The true control variable must be $v_{r}$ that is used in the tap changer definition (see figure 2). However, it is clear that if the following three conditions are respected

- The open-loop control input $n($.$) is piece-wise constant with a sampling period T_{d}$ (where $T_{d}$ is the time delay of the OLTC model)

- The piece-wise constant open-loop control input $n($.$) is such that for all j \in \aleph$, one has

$$
|n(j+1)-n(j)|=d_{n} \quad(=0.02)
$$

where $d_{n}$ is the step size modulus applied to $n$ by the OLTC model when entering the "action" mode.

- The piece-wise constant open-loop control input $n($.$) is such that for all j \in \mathfrak{\aleph}$, one has

$$
(0.8=) \quad n_{\min } \leq n(j) \leq n_{\max } \quad(=1.2)
$$


then it is possible to use $n$ as a constrained control variable and to obtain $v_{r}$ by inverting the dynamic of $n$. This is done by taking for all $t \in\left[j T_{d},(j+1) T_{d}\right.$ [

$$
v_{r}(t)=v(t)-\Gamma \Delta \operatorname{sgn}(n(j+1)-n(j))
$$

where $\Gamma$ is any constant greater than 1 and $\operatorname{sgn}(\cdot)$ is the usual sign function.

Definition 1. A sequence $\{n(j)\}_{j \in \mathbb{N}}$ that meets the above three requirements is said to be tap-changer compatible.

\section{THE NONLINEAR PREDICTIVE CONTROLLER}

Nonlinear predictive control [23] is now widely recognized to be a feedback strategy providing a relatively easy handling of both nonlinearities, constraints and optimality concerns. Recall that predictive control schemes amount to compute at each sampling time an optimal open-loop control sequence (in the sense of some given cost function), to apply the first part of the resulting optimal open-loop control sequence until the next sampling instant. At the next sampling instant, the whole problem is re-considered on a moving-horizon and the procedure is repeated indefinitely resulting in a state feedback law. For nonlinear systems and for long prediction horizons, this may lead to open-loop optimal control problems with a high dimension of the decision variable. This together with the non convex nature of the problem may render the on-line computations necessary to implement the resulting strategy unfeasible. That is the reason why a key feature in nonlinear predictive control design lies in the choice of control parametrization that leads to reasonably reduced complexity. The aim of the following subsection is to clearly define a reduced dimensional parametrization of open-loop controls that are used afterward in the predictive control implementation. Recall that a key feature in receding-horizon control is that the resulting closed-loop control is much more rich than the underlying open-loop parametrization. The consequence of this is that in many cases, apparently over-simplified open-loop parameterizations results in a sufficiently rich closedloop control behavior.

\subsection{The open-loop control parameterization}

Recall that the control is implemented in a sampling scheme with a sampling period $\tau=T_{d}$ and that based on this assumption, the control input in our problem is given at each sampling instant $j \tau\left(\tau=T_{d}\right)$ by

$$
\begin{aligned}
& u(j):=(n(j) b(j)(j))^{T} \\
& \quad \in \quad\left\{n(j-1)-d_{n}, n(j-1),(j-1)+d_{n}\right\} \times \mathrm{B} \times \mathrm{K}
\end{aligned}
$$

with the constraint $n_{\min } \leq n(j) \leq n_{\max }$. Recall that the sets $\mathrm{B}$ and $\mathrm{K}$ have been defined in (6) and (5) respectively.

Remark 1. It is needless to say that the sampling period $\tau$ may be different for the three control components. This enables $k$ and $b$ to be more reactive than $n$ restricted by the tap changer dynamics. In this study, we preferred to adopt a unified sampling scheme for simplicity.

Like any predictive control scheme, one must first define a prediction horizon, that is, the future time horizon over which some cost function is to be minimized. Given some sampling time, this prediction horizon may be defined as an integer number $N_{p}$ of sampling periods. To define the control parametrization, one has to clearly define at each sampling instant $j \tau$ the structure of the control $u(\cdot)$ over the time horizon $\left[j \tau,\left(j+N_{p}\right) \tau\right]$. Before, let us use the following notations to denote the open-loop control profiles at instant $j \tau$ over the prediction horizon $\left[j \tau,\left(j+N_{p}\right) \tau\right]$ 


$$
\begin{aligned}
\widetilde{u}(j) & :=\left(u(j \tau), \ldots, u\left(\left(j+N_{p}-1\right) \tau\right)\right) \\
\widetilde{n}(j) & :=\left(n(j \tau), \ldots, n\left(\left(j+N_{p}-1\right) \tau\right)\right) \\
\widetilde{b}(j) & :=\left(b(j \tau), \ldots, b\left(\left(j+N_{p}-1\right) \tau\right)\right) \\
\widetilde{k}(j) & :=\left(k(j \tau), \ldots, k\left(\left(j+N_{p}-1\right) \tau\right)\right)
\end{aligned}
$$
horizon

The open loop control parametrization is defined by adopting the following choices over the prediction

- The control profiles $\tilde{k}(j)$ and $\tilde{b}(j)$ are constant.

- The control profiles $\tilde{n}(j)$ is monotonic starting from $n(j-1)$

- The control profiles $\widetilde{n}(j)$ is constant on $\left[\left(j+N_{c}\right) \tau,\left(j+N_{p}\right) \tau\right]$ where $N_{c}$ is the control horizon [22].

The last two points define $2 N_{c}+1$ possible choices for $\widetilde{n}(j) . N_{c}$ increasing profiles, $N_{c}$ decreasing profiles and 1 constant profiles. As a result, the resulting parametrization leads to a decision variable of dimension

$$
\left(2 N_{c}+1\right) \cdot \operatorname{card}(\mathrm{B}) \cdot \operatorname{card}(\mathrm{K})
$$

Note also that all candidate sequences $\widetilde{n}(j)$ defined by the above rules are tap changer-compatible in the sense of definition 1. Next and for simplicity the control horizon $N_{c}$ is taken equal to the prediction horizon $N_{p}$. The impact of such is not important since the control profiles are taken constant for the two first control inputs.

\subsection{Definitions and notations}

In order to properly present the optimal control computation, some further definitions and notations are needed. Let $\widetilde{U}(n(j-1))$ be the set of admissible profiles at instant $j \tau$ which were defined before. The fact that this set depends on the past value of $n$ results from (8). This will be shortly denoted by $\widetilde{U}_{j}$. Sometimes, the index $j$ is omitted when no ambiguity follows. We shall define an equivalence relation on $\widetilde{\mathrm{U}}_{j}$ by

$$
\left\{\tilde{u}^{(1)} \sim \tilde{u}^{(2)}\right\} \Leftrightarrow\left\{\left(\tilde{b}^{(1)}, \tilde{k}^{(1)}\right)=\left(\tilde{b}^{(2)}, \tilde{k}^{(2)}\right)\right\}
$$

The need for such equivalence relation comes from the fact that while constraints are imposed on the use of capacitor changes $(b)$ or load shedding $(k)$, no explicit constraint is considered to state that such tap changer compatible sequence $\widetilde{n}^{(1)}$ is better or worst that some other one, still compatible sequence $\widetilde{n}^{(2)}$.

Denote by

$$
\mathrm{U}_{j}^{e q}:=\left\{\widetilde{\mathrm{U}}_{j}^{\left(b^{*}, k^{*}\right)}\right\}_{\left(b^{*}, k^{*}\right) \in \mathrm{B} \times \mathrm{K}}
$$

the set of equivalence classes that partitions $\widetilde{U}_{j}$ according to the equivalence relation (9), namely, an equivalent class (an element of $\left.\mathrm{U}_{j}^{e q}\right)$ is defined by the corresponding $\left(b^{*}, k^{*}\right)$ pair where ones $(r, s)$ denotes an $r \times s$ matrix with all its elements at 1

$$
\widetilde{\mathrm{U}}_{j}^{\left(b^{*}, k^{*}\right)}:=\left\{\widetilde{u}=(\widetilde{n}, \widetilde{b}, \widetilde{k}) \in \widetilde{\mathrm{U}}_{j} \quad \mid \quad \tilde{b}=b^{*} \times \operatorname{ones}\left(1, N_{p}\right) \quad \text { and } \quad \tilde{k}=k^{*} \times \operatorname{ones}\left(1, N_{p}\right)\right\}
$$

Let 


$$
\mathrm{O}: \mathrm{B} \times \mathrm{K} \rightarrow\{1, \ldots, \operatorname{card}(\mathrm{B}) \cdot \operatorname{card}(\mathrm{K})\}
$$

be an ordering of $U_{j}^{e q}$ that reflects the priority in the choice of the control action. Namely

- $\left\{k^{(1)}>k^{(2)}\right\} \Rightarrow\left\{\mathrm{O}\left(b^{(1)}, k^{(1)}\right)>\mathrm{O}\left(b^{(2)}, k^{(2)}\right)\right\}$

- $\quad\left\{k^{(1)}=k^{(2)}\right.$ and $\left.b^{(1)}>b^{(2)}\right\} \Rightarrow\left\{\mathrm{O}\left(b^{(1)}, k^{(1)}\right)>\mathrm{O}\left(b^{(2)}, k^{(2)}\right)\right\}$

This order relation reflects the concern that changes in the capacitors have to be preferred rather than load shedding if the control $n$ is unable to lonely recover the voltage collapse. We shall denote by $X\left(t ; x_{0}, \tilde{u}\right)$ the solution $x(\cdot)$ of the system's equations at time $t$ starting from the initial conditions $\left(0, x_{0}\right)$ and under the control sequence $\tilde{u}$. Using this notation, the following two admissible control profiles are defined (subscript "nc" is used for no collapse)

$$
\begin{aligned}
& \mathrm{U}_{n c}\left(x_{0}\right):=\left\{\tilde{u} \in \widetilde{\mathrm{U}}_{j}^{\left(b^{n c}, k^{n c}\right)} \quad \mid\left(b^{n c}, k^{n c}\right)=\arg \min _{\left(b^{*}, k^{*}\right) \in B \times K} \mathrm{O}\left(b^{*}, k^{*}\right):\right. \\
& \left.X\left(\cdot, x_{0}, \tilde{u}\right) \text { is defined over }\left[0, N_{p} \tau\right]\right\}
\end{aligned}
$$

in other words, $\mathrm{U}_{n c}\left(x_{0}\right)$ is the set of open loop control profiles such that starting from $x_{0}$, no voltage collapse occurs on the prediction time interval $\left[0, N_{p} \tau\right]$. Finally, the following set needs to be defined

$$
\begin{aligned}
& \mathrm{U}_{f}\left(x_{0}\right):=\left\{\tilde{u} \in \widetilde{\mathrm{U}}_{j}^{\left(b^{f}, k^{f}\right)} \mid \quad\left(b^{f}, k^{f}\right)=\arg \min _{\left(b^{*}, k^{*}\right) \in B \times K} \mathrm{O}\left(b^{*}, k^{*}\right):\right. \\
& \left.y_{i}\left(N_{p} \tau, x\left(N_{p} \tau\right), \tilde{u}\right) \geq \underline{y}_{i}, i \in\{1,2,3\}\right\}
\end{aligned}
$$

This is the set of controls for which the resulting near steady state voltage meets the viability requirement. The last inequalities are component wise constraints.

\subsection{The implicit feedback law}

Having at hand the notations of the preceding section, the proposed nonlinear predictive control may be properly defined. The "optimal open-loop" control profile at instant $j \tau$ is obtained by solving the following optimization problem

$$
\hat{u}(x(j), j)= \begin{cases}\arg \min _{\widetilde{u} \in U_{f}} J(\tilde{u}, x(j), j) & \text { if } \mathrm{U}_{f} \neq \varnothing \\ \arg \min _{\widetilde{u} \in U_{n c}} J(\tilde{u}, x(j), j) & \text { if } \mathrm{U}_{f}=\varnothing\end{cases}
$$

where

$$
J(\widetilde{u}, x(j), j):=\int_{0}^{N} p^{\tau}\left(y(\tau, x(j), \widetilde{u})-y_{r e f}\right)^{T} Q\left(y(\tau, x(j), \widetilde{u})-y_{r e f}\right) d \tau
$$

and $y_{\text {ref }}$ is a reference signal and $Q$ is a weighting matrix. The optimization leads to the following sequence of inputs

$$
\hat{u}(x(j), j)=\left(\hat{u}_{0}(x(j), n(j-1)) \ldots \hat{u}_{N_{p}-1}(x(j), n(j-1))\right)
$$

where only the first component is applied. Thus leading to the following implicit state feedback

$$
u(t)=\hat{u}_{0}(x(j), n(j-1)) \quad ; \quad \forall t \in[j \tau,(j+1) \tau)
$$

in accordance with the predictive control principle.

\section{SIMULATION RESULTS}

In what follows see figures 7 and 8 are shown the results of the simulations for the case where the prediction horizon is $N_{p}=1$ (a one sampling period). This induces a chattering in the control inputs, more 

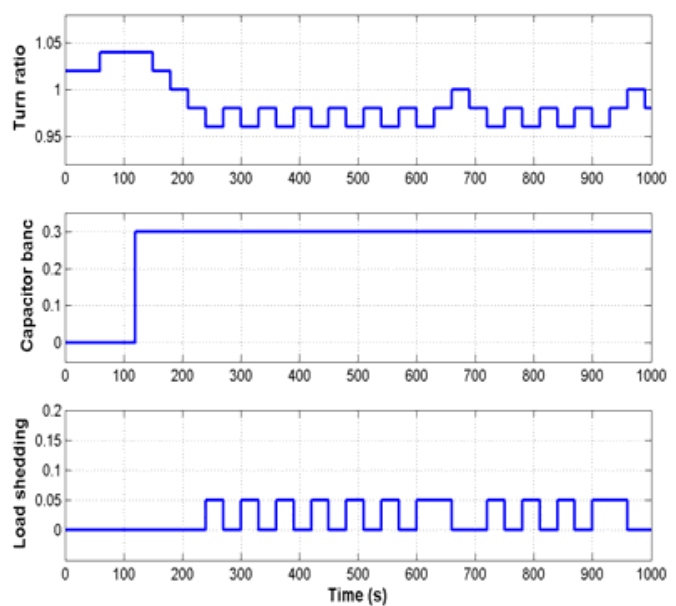

Figure 7. Bus voltages and load states for $N_{p}=1$
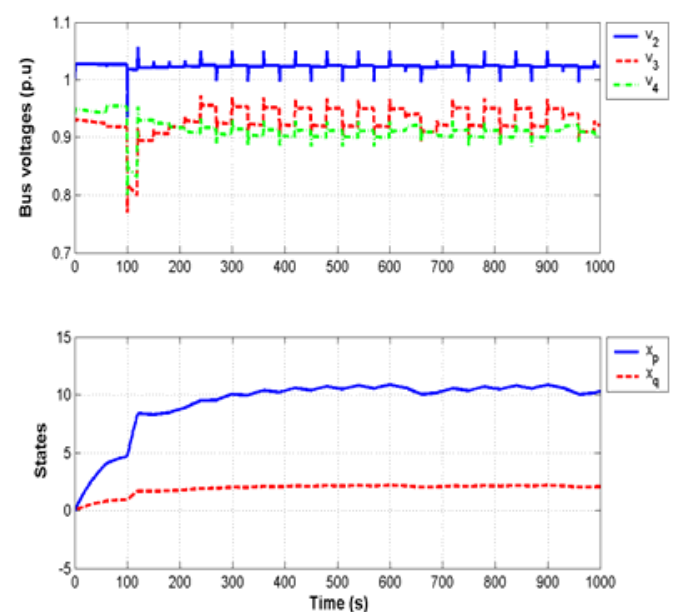

Figure 8. Control inputs for $N_{p}=1$

precisely in the load shedding control input. From a practical viewpoint the chattering of the different control inputs is not acceptable since it drastically reduces the life span of the different control component. This chattering can be alleviated by using a longer prediction horizon. Indeed, a prediction horizon of $N_{p}=1(30 \mathrm{sec})$ does not essentially captures the dominant dynamics of the system since the load time constants are $T_{p}=T_{q}=60 \mathrm{sec}$. For a prediction horizon of $N_{p}=5(150 \mathrm{sec})$ the chattering is suppressed. The voltage responses are shown together with the control inputs in respectively figures 9 and 10. The results obtained here (for $N_{p}=5$ ) are exactly the same as those obtained in [31]. The authors in [31] use the Mixed Logical Dynamical Framework [32] to approximate and convert the power system dynamics into a piecewise affine system. As pointed, this led to sensitivities both in the modelling and control phases, moreover the approach proposed is not fully systematic in the sense that a heuristic value of the capacitor is used. The problem is thus simplified by using as control only the transformer turn ratio. In figures 11-12 are depicted the voltage, states and control input corresponding to one of the most difficult scenarios. Indeed this has been identified as difficult based on an analysis carried out on a simple two nodes system, see [33]. This analysis revealed that for the case of low initial values of the transformer turn ratio, the steady state voltages correspond to the nearest equilibria to the singular surface (the surface described by the algebraic load flow equations). As may seen from these figures, chattering is also present for the case where $N_{p}=1$. In figures 13-14 the same scenario is tested for the case where $N_{p}=5$. The chattering is thus avoided but the capacitor bank is activated before the occurrence of the fault $\left(t_{\text {fault }}=100 \mathrm{sec}\right)$. This is due to the fact that the horizon of $N_{p}=5$ remains shorter and the controller will not be aware that the sole action of the transformer turn ratio is sufficient to stabilize the voltages at a higher level. Load shedding is also used in the transient phase just after the fault detection. In order to avoid such a useless activation of the capacitor, a longer prediction horizon should be used. In figures 1516 are depicted the system responses for $N_{p}=10$. The capacitor is used only after fault detection, a load shedding of $5 \%$ is introduced for approximately more than $2 \mathrm{~min}$. In figure 17 is plotted the computation time versus the prediction horizon. Despite the facts that the controller is implemented on one of the highest level programming languages (Matlab) and that it spends most of its time getting simulations from the power system model (converted from Dymola to Simulink). With all these in mind a prediction horizon up to $N_{p}=30$ can be implemented. In real time applications and for larger scale systems by using more advanced simulations techniques, one can hope to implement this strategy with comparable prediction horizons. Although, for the case study and beyond $N_{p}=5$ no performance improvement is achieved. The 

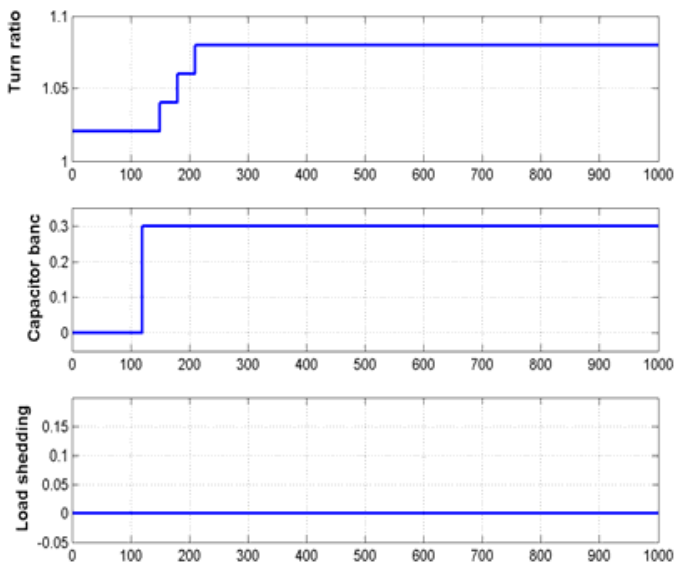

Figure 9. Bus voltages and load states for $N_{p}=5$
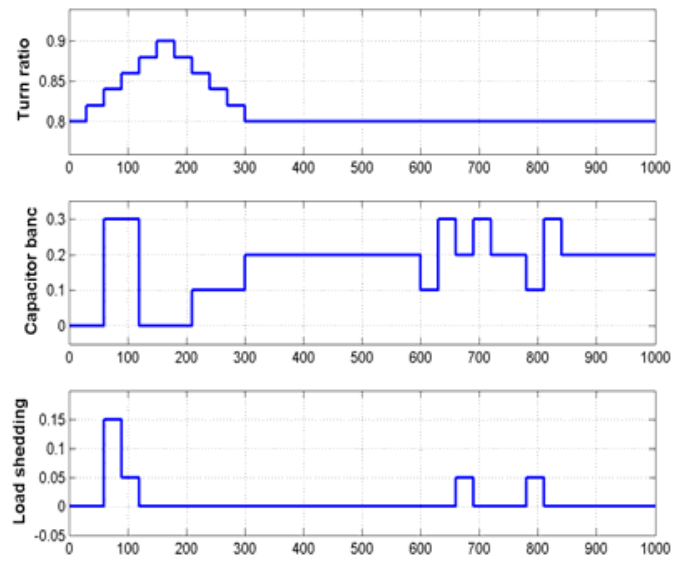

Figure 11. Bus voltages and load states for $N_{p}=1$, $n(0)=0.8$
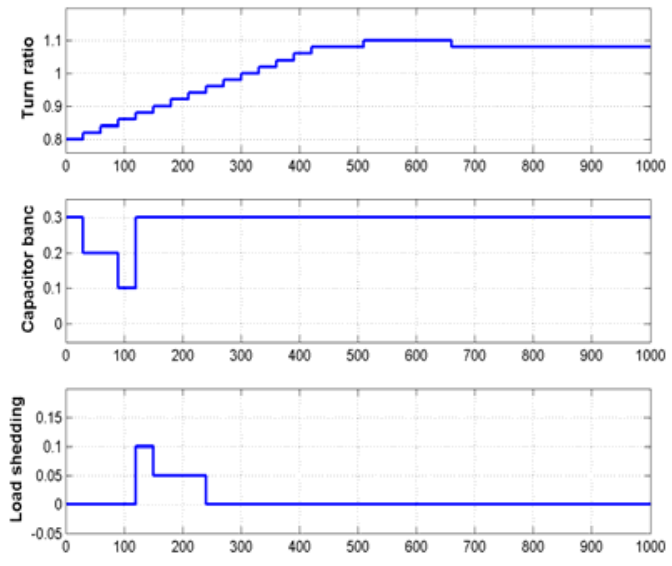

Figure 13. Bus voltages and load states for $N_{p}=5$, $N_{p}=5, n(0)=0.8$
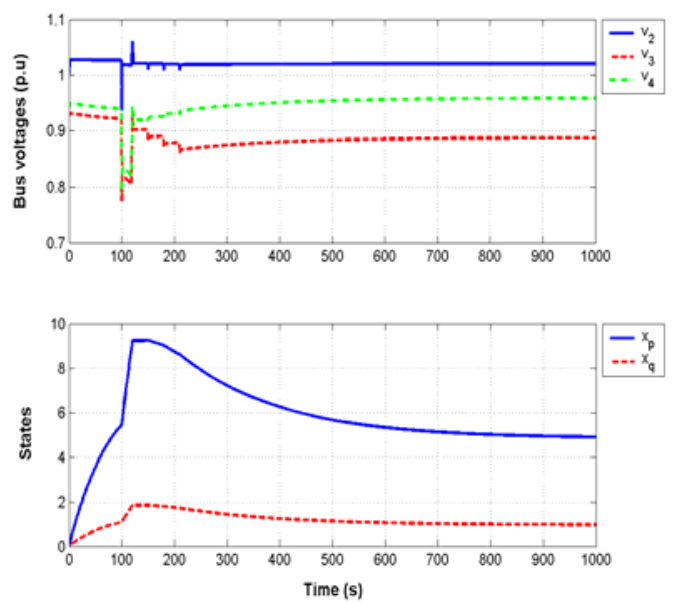

Figure 10. The control inputs for $N_{p}=5$
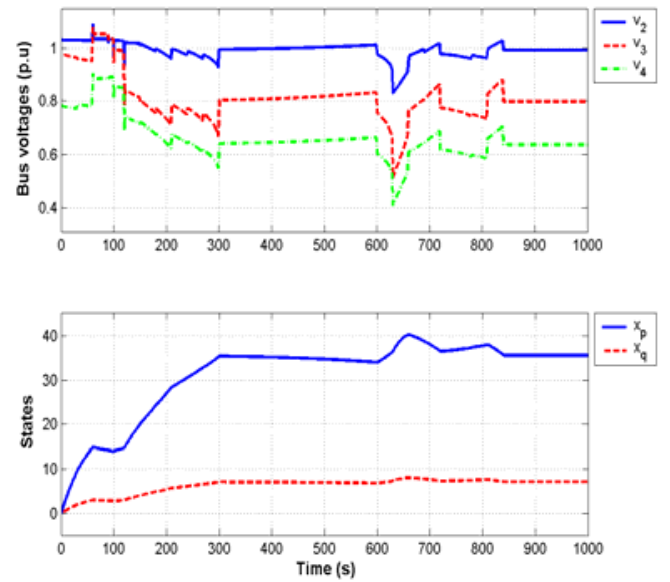

Figure 12. The control inputs for $N_{p}=1$, and $n(0)=0.8$
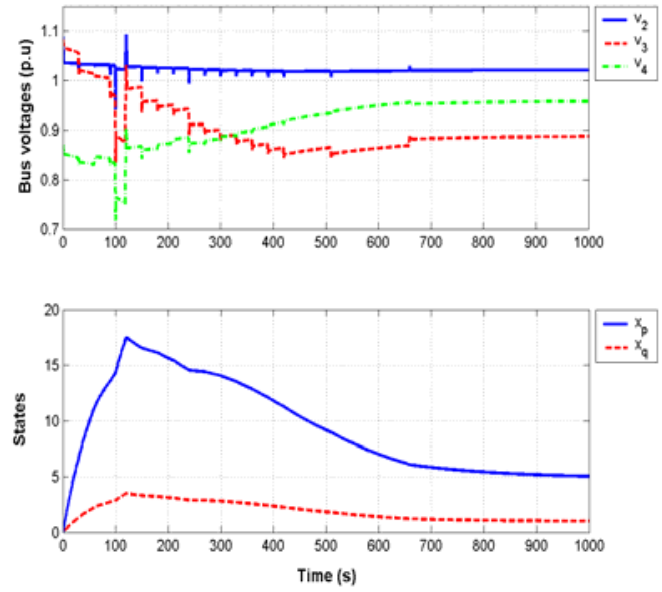

Figure 14. The control inputs for and $n(0)=0.8$ 

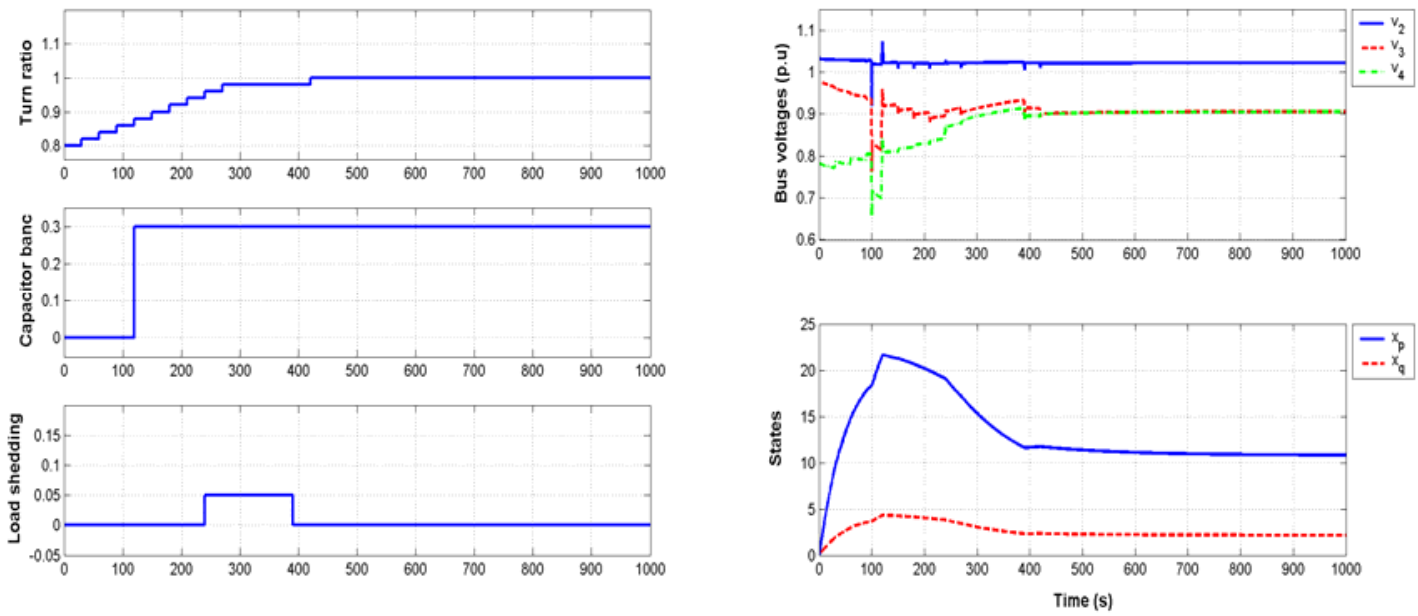

Figure 15. Bus voltages and load states for $N_{p}=5$ $N_{p}=5$ and a delay of and a delay of $110 \mathrm{sec}$

Figure 16. The control inputs for $110 \mathrm{sec}$

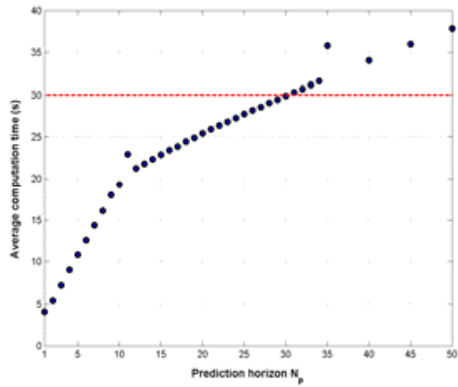

Figure 17. The computation time versus $N_{p}$

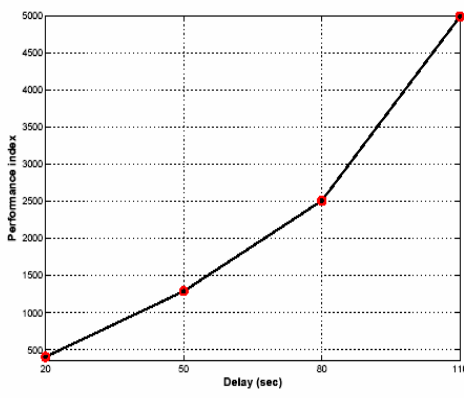

Figure 18. Performance versus $N_{p}$

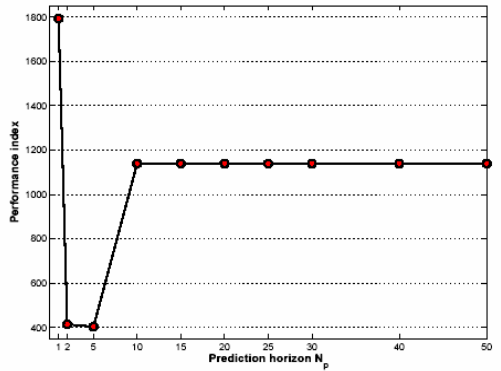

Figure 19. Performance versus the delay
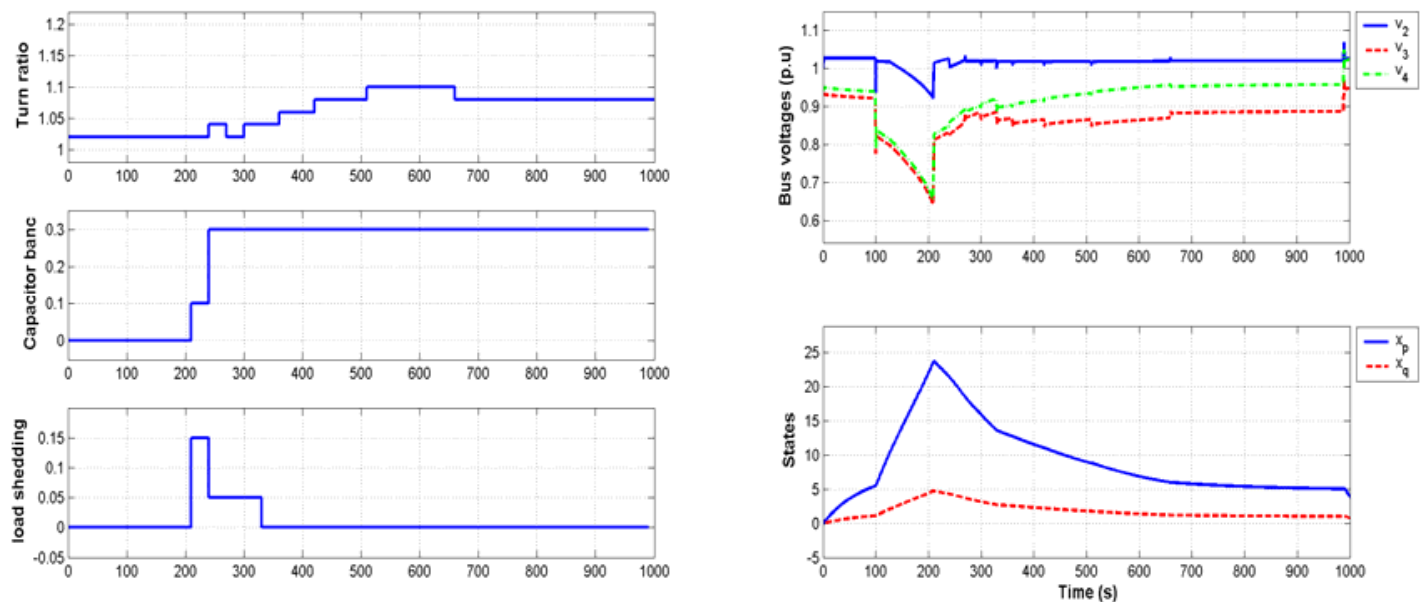

Figure 20. Bus voltages and load states for $N_{p}=10$, Figure 21. The control inputs for $N_{p}=10$, $n(0)=0.8$ and $n(0)=0.8$ 
performance is worsened, we conclude that the optimum prediction horizon for the system under nominal conditions $\left(n(0)=1.02\right.$ and a delay fault-action $\tau_{d}$ of $\left.20 \mathrm{sec}\right)$ is 5 , see figure 18 . In order to show the importance of a dynamic analysis. The delay $\tau_{d}$ between the fault occurrence and its detection is varied, see figure 19. For a delay of about $140 \mathrm{sec}$ an acceptable voltage level can not restored and voltage collapse is inevitable under the actual constraints on the different control inputs. This is closely related to critical time approaches, [34]. For instance, in figure 20-21 are plotted the different variables when the delay is $110 \mathrm{sec}$. Although, the steady state inputs are the same as in the preceding cases (static analysis see figure 6) load shedding is used in the transient phase, this is in accordance with the fact that delaying too much the action leads one to shed more load (if reaction is made instantaneously the steady states values are sufficient) see e.g., [10] where the same conclusions are drawn. Recall this type of control behavior can not be inferred from the static analysis.

\section{CONCLUSIONS}

In this paper a predictive control approach is developed for voltage stabilization of power systems. Despite its tuning easiness, the approach is shown to be computationally efficient which makes it attractive for real time applications. Moreover, numerous simulation scenarios testify the viability of such an approach. By using suboptimal search methods, extension to large scale systems can be made possible within the computational time restriction. This is currently an issue under investigation, and some promising results are available.

\section{REFERENCES}

1. W. H. Esselman, et al., "Hybrid discrete and continuous control for power systems", Discrete Event Dynamic Systems, 9, pp. 297-318, 1999.

2. P. J. Antsaklis and A. Nerode, Special issue on hybrid systems, IEEE Trans. On Aut. Control, Vol. 43, $\mathrm{N}^{\circ} 4,1998$.

3. S. Morse, et al., Special issue on hybrid systems, Automatica, vol. 35, № 3, 1999.

4. P. J. Antsaklis, Special issue on hybrid systems, Proc. of the IEEE, vol. 88, N7, 2000.

5. $\quad$ P. Fairley, "The unruly power grid", IEEE Spectrum, 2004.

6. T. Van Cutsem, "Voltage instability: Phenomena, countermeasures and analysis methods", Proc. of the IEEE, vol. 88, N², pp. 208-227, 2000.

7. K. T. Vu, et al., "Voltage instability: Mechanisms and control strategies", Proc. of the IEEE, vol. 83, No 11, pp. 1442-1455, 1995.

8. IEEE/CIGRE Joint Task Force on Stability Terms and Definitions, "Definition and classification of power system stability", Technical report, 2004.

9. I. A. Hiskens, "Analysis tools for power systems- Contending with nonlinearities", Proc. of the IEEE, Vol. 83, № 11, pp. 1573-1587, 1995.

10. T. Van Cutsem, et al., "Design of Load shedding schemes against voltage instability using combinatorial optimization", IEEE PESW Meeting, 2002.

11. C.A. Canizares, et al., "Comparison of performance indices for detection of proximity to voltage collapse", IEEE Trans. on Power Systems, vol. 11, N³, pp. 1441-1450, 1996.

12. Q. Wu, et al., "Voltage security enhancement via coordinated control", IEEE Trans. on Power Systems, vol. 16, $\mathrm{N}^{\circ} 1$, pp. 127-135, 2001.

13. T. Van Cutsem, et al., "A comprehensive analysis of mid-term voltage stability", IEEE Trans. on Power Systems, vol. 10, №3, pp. 1173-1182, 1995.

14. M. La Scala, et al., "On line dynamic preventive control: an algorithm for transient security dispatch", IEEE Trans. on Power Systems, vol. 13, º2, pp. 601-610, 1998.

15. A. Takehara, et al., "Voltage stability preventive and emergency-preventive control using VIPI sensitivity", Electrical Engineering in Japan, vol. 143, N 4, pp. 22-30, 2003.

16. Z. Feng, et al., "A Comprehensive approach for preventive and corrective control to mitigate voltage collapse”, IEEE Trans. on Power Systems, vol. 15, №2, pp. 791-797, 2000.

17. I. Hiskens and M. A. Pai, "Trajectory Sensitivity Analysis of Hybrid Systems", IEEE Trans. on Cir. and Sys., vol.47, N², pp.204-220, 2000. 
18. J. Y. Wen, et al., "Optimal Coordinated Voltage Control for Power System Voltage Stability", IEEE Trans. on Power Systems, vol. 19, №2, pp. 1115-1122, 2004.

19. M. Larsson and D. Karlsson, "Coordinated System Protection Scheme Against Voltage Collapse Using Heuristic Search and Predictive Control”, IEEE Trans. on Power Systems, vol. 18, N³, pp. 1001-1006, 2003.

20. D. Q. Mayne and H. Michalska, "Receding horizon control of nonlinear systems", IEEE Trans. on Aut. Cont., vol.35, ํ7, pp. 814-824, 1990.

21. W. H. Chen, et al., "Model predictive control of nonlinear systems: computational burden and stability", IEE Proc. On Cont. The. and Appl., vol.147, №4, pp. 387-394, 2000.

22. J. B. Rawlings, "Tutorial overview of model predictive control", IEEE Cont. Sys. Magazine, vol. 20, $\mathrm{N}^{\circ} 3$, pp. 38-52, 2000.

23. D. Q. Mayne, et al., "Constrained model predictive control: Stability and optimality", Automatica, vol.36, pp.789-814, 2000.

24. M. Larsson and P. Korba, "Benchmark Model for Hybrid Systems Design based on a small-scale Power System Model", This issue.

25. B. Fardanesh, "Future trends in power system control", IEEE Comp. Appl. in Power, pp. 24-31, 2002.

26. T. Nagata and H. Sasaki, "A multi-agent approach to power system restoration”, IEEE Trans. on Power Systems, vol. 17, №2, pp. 457-462, 2002.

27. H. Ni, et al., "Power system stability agents using robust wide area control", IEEE Trans. on Power Systems, vol. 17, №4, pp. 1123-1131, 2002.

28. V. Venkatasubramanian, et al., "A Taxonomy of the Dynamics of Large Constrained Nonlinear Systems", Proc. of the IEEE, vol.83, №11, pp. 1530-1561, 1995.

29. J. Zaborsky and M.D. Ilic, Dynamics and Control of Large Electric Power Systems, Wiley Interscience, 2000.

30. J. A. Momoh, et al., "Challenges to optimal power flow", IEEE Trans. on Power Systems, vol. 12, $\mathrm{N}^{\circ} 1$, pp. 444-455, 1997.

31. T. Geyer, et al., "Hybrid Emergency voltage control in power systems", Proceedings of the European Control Conference Oxford, UK, 2003.

32. A. Bemporad and M. Morari, "Control of Systems Integrating Logic, Dynamics, and Constraints", Automatica, vol.35, pp. 407-427, 1999.

33. M. Larsson, "A Simple Test System Illustrating Load Voltage Dynamics in Power Systems", Technical report $A B B, 2002$.

34. L. Varga and C. Canizares, "Time dependence of controls to avoid voltage collapse", IEEE Trans. on Power Systems, vol. 15, ํ4, pp. 791-797, 2000.

\section{ABOUT THE AUTHORS}

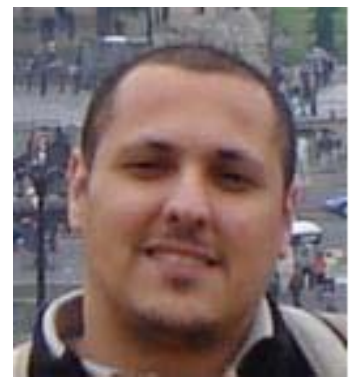

S. A. Attia received an engineer diploma in Electrical engineering (power components) from the university USTO, Oran in 1999. After spending two years as an associate researcher at the same university, He joined the LAG (Laboratoire d'Automatique de Grenoble), Polytechnic of Grenoble, where He obtained the Msc in Automatic control. He is now a Phd candidate at the same department. His research interests are in the field of nonlinear optimal control with emphasis on the computational aspect and applications. 
M. ALAMIR graduated in Mechanical Engineering from the Polytechnic of Grenoble (INPG), France (1990) and in Aeronautics from the Ecole Nationale SupXerieure des IngXenieurs en Construction Aéronautiques (ENSICA), France (1992). He received a Ph.D. from INPG in Nonlinear Control (1995). Since 1996, he has been a research associate CNRS in the Laboratoire d'Automatique de Grenoble in Saint Martin d'Hères, France. His main research interests are nonlinear receding horizon control, nonlinear receding horizon observers, robust nonlinear and optimal control as well as industrial control applications.
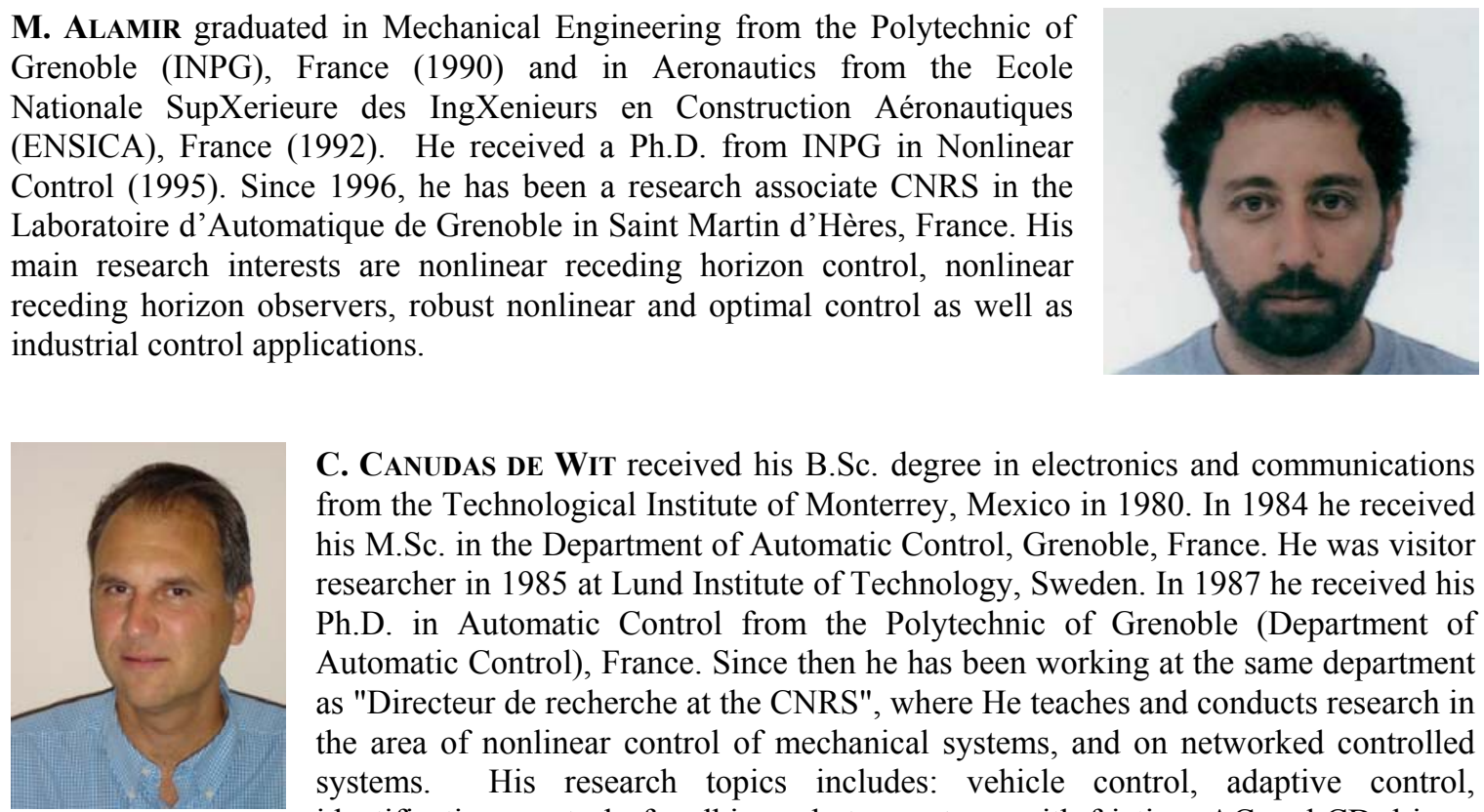

C. CANUdas DE WIT received his B.Sc. degree in electronics and communications from the Technological Institute of Monterrey, Mexico in 1980. In 1984 he received his M.Sc. in the Department of Automatic Control, Grenoble, France. He was visitor researcher in 1985 at Lund Institute of Technology, Sweden. In 1987 he received his Ph.D. in Automatic Control from the Polytechnic of Grenoble (Department of Automatic Control), France. Since then he has been working at the same department as "Directeur de recherche at the CNRS", where He teaches and conducts research in the area of nonlinear control of mechanical systems, and on networked controlled systems. His research topics includes: vehicle control, adaptive control, identification, control of walking robots, systems with friction, $\mathrm{AC}$ and $\mathrm{CD}$ drives, and networked controlled systems. He has established several industrial collaboration projects with major French companies (FRAMATOME, EDF, CEA, IFREMER, RENAULT, SCHNEIDER, ILL). He has written a book in 1988 on adaptive control of partially known systems: theory and applications (Elservier Publisher). He has also edited a book in 1991 on Advanced Robot Control (Springer-Verlag), a joint 12 author book "Theory of robot control", on Springer Control and Communication Series, in (1997). He has been associate editor of the IEEE-Transaction on Automatic Control, from Jan 1992, to Dec. 1997, and of AUTOMATICA, from 1999, to 2002. His research publications includes: more than 120 International conference papers and more than 47 published papers in international journals. He has supervised $20 \mathrm{Ph}$. D. students. 